\title{
Improving Sleep with the Unique and the Newly Developed Acupoint Protocol to Fight Against Cancer Pain
}

\author{
Tony Hong* \\ As-You-Wish Healthcare Institute, Taiwan \\ *Corresponding author: Tony Hong, As-You-Wish Healthcare Institute, Freelance, Taiwan. \\ To Cite This Article: Tony Hong. Improving Sleep with the Unique and the Newly Developed Acupoint Protocol to Fight Against Cancer Pain. Am \\ J Biomed Sci \& Res. 2019 - 3(1). AJBSR.MS.ID.000624. DOI: 10.34297/AJBSR.2019.03.000624
}

Received: April 23, 2019 | Published: May 14, 2019

\begin{abstract}
This unique and newly developed protocol composed of Yuan-Source and Luo-Connecting acuoints was conducted to the patient at stage IV with urothelial carcinoma. The therapeutic effects of acupuncture show that the patient's pain was quickly reduced from level 9 down to level 4 with the four treatments, enabling him to have the better quality of life with sound sleep. From perspective of the cancer pain management, the pain scale answered by the patient confirmed the pain can be reduced/relieved with the quality of sleep. However, how to get the whole picture of the mechanism of this protocol needs more scientific evidence.
\end{abstract}

Keywords: Yin yang; Cancer pain management; Yuan source/Luo connecting; Acupoints

\section{Introduction}

Pain is a very common condition which can affect the quality of life of patients at any time. Rated from a dull ache to a sharp stab and ranged from mild to server in the daily life, pain management is attracting more and more attention because the occurrence of pain rises when people are getting older. Compared to men, women are more likely to experience pain which is generally classified into acute pain which is understood to be the normal response to tissue injury, and chronic (ongoing) pain that persists beyond the normal time of healing and generally lasts for more than three months [1].

Yin-Yang is the abstract concept in the traditional Chinese medicine (TCM) and acupuncture theories, though it is difficult and plays the key role for the public to catch the whole picture of the mechanism of the TCM and acupuncture. However, scientific evidence shows it still serves as the foundation of pattern for diagnosis and the best treatment outcomes [2].

Cancer pain is one of the medical conditions identified by the WHO at present that can be treated effectively with acupuncture, even though the efficacy of acupuncture treatment still remains a controversial issue worldwide [3]. In face of the opioid crisis, acupuncture, the ancient Chinese form of alternative medicine and primarily used for pain management, has been growing in popularity in the United States and gained support from the U.S. Department of Veterans Affairs and Medicaid as a viable treatment in the recent years. [4,5].
Current research suggests that sleep problems are closely related to the chronic pain. Those people who have the chronic pain frequently find it difficult to fall asleep or sleep poorly that is mostly disrupted with frequent and long night awakenings [6].

This article introduces the newly developed but unique acupoint protocol that can be effective for improving sleep as a solution to fighting against pain.

\section{Introduction to the Protocol}

Yi-Yang, the unique and philosophical concept, serves as the foundation and guideline for developing acupuncture and traditional Chinese medicine (TCM) theories like Qi-Blood, pattern identification, and Five Elements. However, it deserves attention because the imbalance of Yin and Yang can result in a disease. For treatment, the principles of selecting acupoints in the clinical practice are often choosing interiorly-exteriorly related channels, the channels corresponding to Zhang and Fu, Ying-Yang pairing channels, the Yuan-Source acupoints, Collecting acupoints, the FiveShu acupoints, Back-Shu acupoints, the Mother-Child acupoints, and the Front-Mu acupoints, etc. [7].

In acupuncture theories, acupoints are specifically chosen sites that can gather Qi to transport Blood throughout the body. Various therapeutic benefits occur with the stimulation on different acupoints on the body surface. 
Each of the twelve regular channels has a Yuan-Source acupoint to pool the Yuan (original) Qi and a Luo-Connecting acupoint which functions for connecting Zang and Fu organs bearing the exteriorinterior relationship.

It is first discussed and taken into consideration for developing this protocol that Yuan-Source acupoints can be used to treat Zang organs in Chapter one of the Volume of the Spirit Pivot of the Huang Di Nei Jing (Yellow Emperor's Inner Classic) while Fu organs can be treated with Luo-Connecting acupoints in Chapter four.

Yin-Yang balance is the key concept of this new protocol, which consists of both Yuan-Source acupoints treating Zang organs and Luo-Connecting acupoints that can treat $\mathrm{Fu}$ organs in order to reinforce Yang and nourish Yin.

The Yin-Yang theory indicates that the left refers to Yang while Yin lies in the right; therefore, the manipulation procedures of this protocol must follow the steps listed below for the best outcomes.

I. The protocol needs to be conducted four consecutive times to the patients and once per day.
II. On the Yin meridians, Yuan-Source acupoints of Zang organs are selected and Luo-Connecting acupoints of Fu organs on the Yang meridians.

III. Start with Yuan-Source acupoint on the Lung meridian, following the sequence of the flow of Qi, and end on YuanSource acupoint of Liver on the Liver meridian.

\section{Discussion}

Acupuncture has been verified to be beneficial to patients suffering from chronic pain with the clinically relevant effect of acupuncture on chronic pain that can persist over time $[8,9]$

This protocol aimed at reinforcing Yang to nourish Yin was conducted to one of the nine cases, in which the patient, male and 57 years old, who had been diagnosed at Stage IV/cT2N3M1 five months ago as diabetes, infiltrating urothelial carcinoma, right pelvis, and suspected of lower ureter with thoracic spine metastasis before the treatments. The pain was rated level 10 before the first treatments, even though Fentanyl patches were used for pain relief.

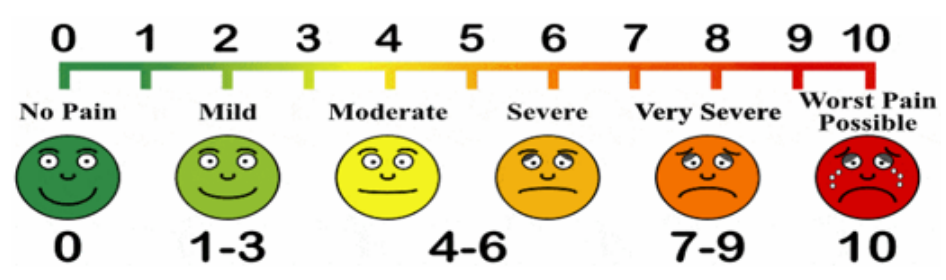

Figure 1: Pain scale used in the treatments

Table 1: Major reactions with pain levels.

\begin{tabular}{|c|c|c|}
\hline Tx & Major reactions & Level of pain \\
\hline 1 & Sleep improved a little bit and bowel movement at 1 a.m. with the treatment at 8 p.m. & Level 8 \\
\hline 2 & Appetite improved to be able to eat a bowl of needles at 9:30 p.m. with reduced pain. & Level 6 \\
\hline 3 & Felt calm to sleep soundly. Uses of Fentanyl patches decreased from 4 to 2 patches & Level 4 \\
\hline 4 & Patient could stand to open the door and speak with the normal voice. Stopped using Fentanyl patches. & Level 3 \\
\hline
\end{tabular}

The result showed that the levels of pain rated on the WongBaker FACES Pain Scale as Figure 1 by the patient decreased with each treatment as shown in Table 1.
The tongue diagnosis serves as the one of the objective information for evaluation of treatment. The regressed sticky white coating also showed the patient's condition had been improved greatly with the treatments as shown in Figure 2.

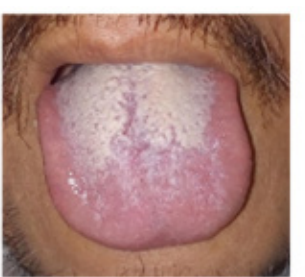

Before

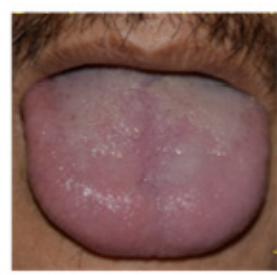

After

Figure 2: Tongue coating after treatments.

Ample research suggests that sleep disturbance may impair key processes contributing to the development and maintenance of chronic pain, which highlights sleep is closely associated with pain [10]. One of the solutions to pain management is acupuncture that has been proved to have beneficial effects as a treatment for improving the quality of sleep [11].
One of the notable results in the past nine cases using this protocol is that all of the patients claimed the quality of sleep was improved ideally in a short time with one or two treatments to reduce pain. In other words, the conclusions in common seems to suggest that the role of sleep is crucial in pain management. 


\section{Conclusion}

Facing the opioid crisis, researchers' interest in acupuncture for pain relief are revived and trying to determine whether or not acupuncture can be a beneficial and alternative treatment for various health ailments.

The past cases show this unique and newly developed acupoint protocol works effectively through activating Yin and Yang at the same time to reduce pain. This case also suggests referral for acupuncture treatment is a reasonable option for patients with chronic pain, particularly for those patients who are seeking solutions to cancer pain.

However, the mechanism of this protocol remains unknown from the aspect of conventional medicine, suggesting that more science-based evidence is required in the future.

\section{References}

1. Pain and pain management - adults. Victory State Government. Better Life Channel.

2. Hong TZ (2018) Cautions for Learning Traditional Chinese Medicine and Acupuncture. Advancements Bioequiv Availab 2(2).

3. Review of Systemic Reviews of Acupuncture for Numberous Conditions. Evidence Based Acupuncture.
4. Ehrenfeld T (2016) Why People are choosing Acupuncture Over Opioids for Pain Relief. Healthline.

5. Jonathan Wilkinson J, Faleiro R (2007) Acupuncture in pain management. Continuing Education in Anaesthesia Critical Care \& Pain 7(4): 135-138.

6. Sleep and pain Learn how you can encourage sleep to form part of your musculoskeletal pain co-management plan. Pain Health, Department of Health.

7. Hong TZ (2018) Decision-Making Model Proposed in Use of Extra/A-shi Acupoints with Traditional Acupoints. Advancements Bioequiv Availab 2(2): $1-3$.

8. Vickers AJ, Vertosick EA, Lewith G, MacPherson H, Foster NE, et al. (2017) Acupuncture for Chronic Pain: Update of an Individual Patient Data Meta-Analysis. The Journal of Pain 19(5): 455-474.

9. Ingraham P (2018) Does Acupuncture Work for Pain? A review of modern acupuncture evidence and myths, focused on treatment of back pain \& other common chronic pains. Pain Science.

10. Finan PH, Goodin BR, Smith MT (2013) The association of sleep and pain: an update and a path forward. J Pain 14 (12): 1539-1552.

11. Reshef A, Bloch B, Vadas L, Ravid S, Kremer I, et al. (2013) The Effects of Acupuncture Treatment on Sleep Quality and on Emotional Measures among Individuals Living with Schizophrenia: A Pilot Study. Sleep Disorders p. 1-11. 